\title{
IDENTIFICAÇÃO DE EXTRATOS ETANÓLICOS DE MADEIRAS UTILIZANDO SEU ESPECTRO ELETRÔNICO DE ABSORÇÃO E ANÁLISE MULTIVARIADA
}

\author{
Alexandre Ataide da Silva, Eduardo Sanches Pereira do Nascimento, Daniel Rodrigues Cardoso e Douglas Wagner Franco* \\ Instituto de Química de São Carlos, Universidade de São Paulo, CP 780, 13560-970 São Carlos - SP, Brasil
}

Recebido em 12/7/11; aceito em 20/9/11; publicado na web em 6/12/11

\begin{abstract}
IDENTIFICATION OF ETHANOLIC WOOD EXTRACTS USING ELECTRONIC ABSORPTION SPECTRUM AND MULTIVARIATE ANALYSIS.The application of multivariate analysis to spectrophotometric (UV) data was explored for distinguishing extracts of cachaça woods commonly used in the manufacture of casks for aging cachaças (oak, cabreúva-parda, jatobá, amendoim and canela-sassafrás). Absorbances close to $280 \mathrm{~nm}$ were more strongly correlated with oak and jatobá woods, whereas absorbances near $230 \mathrm{~nm}$ were more correlated with canela-sassafrás and cabreúva-parda. A comparison between the spectrophotometric model and the model based on chromatographic (HPLC-DAD) data was carried out. The spectrophotometric model better explained the variance data $(\mathrm{PC} 1+\mathrm{PC} 2=91 \%)$ exhibiting potential as a routine method for checking aged spirits.
\end{abstract}

Keywords: spectrophotometric model; fingerprint chromatographic; cachaça wood extract.

\section{INTRODUÇÃO}

Bebidas alcoólicas recém-destiladas em geral apresentam características sensoriais pouco apreciadas por parte dos consumidores. ${ }^{1,2}$ O envelhecimento de bebidas em tonéis de madeira é extensamente empregado pela indústria em função das conhecidas melhorias sensoriais conferidas às bebidas submetidas a este processo. ${ }^{3,4}$ Esta é a razão pela qual o uisque, o rum, o conhaque e a grappa, por exemplo, incluem em seu processo de produção, a etapa de armazenagem em tonéis de madeira. ${ }^{5}$ Em alguns casos, como os do uisque e do conhaque, esta etapa é obrigatória para a sua comercialização. ${ }^{6}$

A aguardente brasileira ou cachaça ${ }^{7,8}$ tem por virtude ser apreciada tanto em sua forma "jovem", "branca" ou "descansada", quanto na forma de aguardente "envelhecida". Em linhas gerais, o processo de envelhecimento da cachaça segue os moldes dos sistemas americano e europeu reutilizando barris de carvalho, ${ }^{9,10}$ oriundos da indústria de bebidas alcoólicas da Europa ou dos Estados Unidos.

A exploração do carvalho no continente europeu mostra sinais de esgotamento ${ }^{11}$ deste recurso vegetal motivando, assim, a busca de novas madeiras como alternativas ao carvalho e procurando manter a qualidade do produto final. Existe, portanto, uma demanda crescente $\mathrm{e}$ declarada para o desenvolvimento da química das madeiras utilizadas para construção de tonéis e também de ferramentas analíticas que possam assegurar a qualidade, a confiabilidade e a rastreabilidade do produto para o consumidor.

O emprego de madeiras tipicas da flora brasileira na confecção de tonéis para o envelhecimento de bebidas dá origem a produtos muito apreciados. ${ }^{9}$ Esta prática se desenvolveu ao longo dos anos seguindo tradições locais e de forma empírica. Isto conduziu a produtos com propriedades sensoriais agradáveis, mas sem a devida avaliação dos possíveis efeitos da ingestão destes extratos sobre a saúde do consumidor. Exemplo deste procedimento é a utilização de tonéis de canela sassafrás, que embora conduza a produto apreciado por suas qualidades sensoriais, passa a apresentar safrol, ${ }^{12}$ como consequência do contato com a madeira, em sua composição. Este composto está banido pela FDA ${ }^{13}$ em alimentos por ser considerado carcinogênico. Extratos alcoólicos de canela sassafrás também apresentam acentuado efeito pró-oxidante. ${ }^{14}$ Estes dois quesitos não recomendam a

*e-mail: douglas@iqsc.usp.br sua utilização na confecção de tonéis. Existe, portanto, a necessidade declarada de avaliar as propriedades sensoriais e os possíveis efeitos sobre a saúde humana do emprego de madeiras brasileiras no envelhecimento de cachaças..$^{914-20}$

A classificação de uma árvore, matéria-prima para a produção de tonéis, é realizada considerando principalmente os seus órgãos reprodutores (flores e frutos) e, também, outras características morfológicas da árvore (casca, folhas, etc.) e mesmo em alguns casos a análise do seu aroma. ${ }^{21} \mathrm{~A}$ aplicação destas metodologias requer considerável experiência e habilidade por parte do analista.

As tentativas taxonômicas no reino vegetal, especificamente no caso das madeiras, não são tão frequentes quanto poderiam ser, empregando técnicas instrumentais associadas à interpretação físico-química dos resultados. ${ }^{22}$ Aos esforços nesta direção somam-se recentemente as aplicações de técnicas quimiométricas a banco de dados provenientes das aplicações de diferentes técnicas instrumentais a extratos de madeiras e de produtos de origem vegetal. ${ }^{16,17}$

Como parte dos esforços para o desenvolvimento da análise química da aguardente, neste trabalho são apresentados resultados sobre a aplicação da técnica espectrofotométrica (UV) com tratamento quimiométrico dos espectros eletrônicos para a caracterização de extratos de 4 espécies de madeiras da flora brasileira e do carvalho. Os dados aqui reportados foram comparados aos resultados obtidos a partir de perfis cromatográficos (CLAE-DAD), anteriormente descritos ${ }^{16}$ para estas amostras, objetivando a elaboração de um procedimento de rotina para a identificação de extratos hidroalcoólicos de madeiras com base na espécie empregada.

\section{PARTE EXPERIMENTAL}

Todos os solventes e reagentes utilizados foram de grau analítico e/ou pureza cromatográfica e adquiridos da Merck (Darmstadt, Alemanha) e da Mallinckrodt (Phillipsburg, NJ, EUA). A água utilizada $\left(18 \mathrm{M} \Omega \mathrm{cm}^{-1}\right)$ foi destilada e posteriormente deionizada em um sistema Milli-Q da Millipore (Bedford, MA, EUA).

\section{Amostras de madeiras}

A amostra de cachaça devidamente certificada pelo produtor, não envelhecida ( $47 \%$ v/v), sem adição de açúcar e utilizada na preparação 
dos extratos, foi gentilmente doada pela Indústria Müller de Bebidas Ltda. (Pirassununga, São Paulo, Brasil). As amostras de amendoim (Pterogyne sp.), jatobá (Hymenaea courbaril), canela sassafrás (Ocotea pretiosa) e cabreúva-parda (Myrocarpus frondosus) foram cedidas pelos Laboratórios de Madeiras e Estrutura de Madeiras da Universidade de São Paulo, São Carlos. As amostras de carvalho (Quercus sp.) devidamente certificadas foram cedidas pelo Prof. J. Piggott do Departamento de Biociências de Strathclyde (Escócia, Glasgow).

\section{Preparo dos extratos}

Seguindo procedimento anteriormente descrito, ${ }^{14,16}$ as amostras de madeiras foram moídas e o pó peneirado de forma a obter partículas de 100 mesh de diâmetro. Estes materiais foram então extraídos com cachaça não envelhecida ( $\mathrm{pH}=4,7 \pm 0,1)$, na proporção $0,010 \mathrm{~g} \mathrm{~mL}^{-1}$. O processo de extração foi realizado durante 26 dias sob agitação constante, à temperatura ambiente $\left(25 \pm 2{ }^{\circ} \mathrm{C}\right)$ e ao abrigo da luz. As amostras, sempre em duplicata, após preparadas foram filtradas em papel de filtro quantitativo e armazenadas à temperatura ambiente. No total foram preparados 18 extratos, sendo 9 de carvalho, 2 de amendoim, 2 de jatobá, 3 de cabreúva-parda e 2 de canela sassafrás. Apesar da grande variedade de madeiras utilizadas para a confecção de tonéis, ${ }^{914-17,20}$ a amostragem ficou restrita ao carvalho, ao amendoim, ao jatobá, à cabreúva-parda e à canela sassafrás, devido a sua reconhecida utilização por parte dos produtores e à existência de dados cromatográficos (CLAE-DAD $)^{16}$ para estas amostras facilitando uma comparação.

\section{Espectros eletrônicos (UV)}

Os espectros eletrônicos de absorção para as amostras, na região de 200 a $400 \mathrm{~nm}$, foram coletados em um espectrofotômetro HP $8152 \mathrm{~A}$ com resolução de $2 \mathrm{~nm}$ e utilizando-se de cubetas de quartzo de caminho ótico de $1 \mathrm{~cm}$ (Hellma, Müllheim, Germany). Com a finalidade de normalizar os resultados, quando necessário as amostras foram diluidas em etanol/água $40 \% \mathrm{v} / \mathrm{v}$ com o objetivo de se ajustar o valor da absorbância obtida em $220 \mathrm{~nm}$ ao valor unitário.

\section{Perfil cromatográfico}

As condições operacionais estão descritas em publicação anterior. ${ }^{16}$ No intuito de facilitar a consulta, estão reproduzidas como Material Suplementar.

\section{Análise multivariada}

Os dados espectrofotométricos depois de normalizados e aplicada primeira derivada ( $\mathrm{dAbs} / \mathrm{d} \lambda$ ) foram utilizados na construção de uma matriz 18 linhas x 90 colunas. As linhas correspondem às 18 amostras e as colunas aos dados de absorbância (220-400 nm a cada $2 \mathrm{~nm}$ ) para cada um dos extratos. A análise multivariada foi realizada utilizando os softwares The Unscrambler V9.8 e MINITAB Statistical Software versão 14 .

O conjunto de dados obtidos foi alinhado para remover eventuais deslocamentos de picos cromatográficos oriundos de pequenas oscilações das condições de análise utilizando o algoritimo $\mathrm{COW}$ (Correlation Optimization Warping), proposto anteriormente. ${ }^{23}$ Foi então realizado alinhamento da linha de base pelo método MSC (multilpe scaterring curve) e construída uma matriz com 18 linhas x 300 colunas. ${ }^{16}$

\section{RESULTADOS E DISCUSSÃO}

Foi observado em estudos sobre tequila, ${ }^{24}$ estarem as bandas próximas a $280 \mathrm{~nm}$ relacionadas à presença de compostos orgânicos e traços de voláteis provenientes do processo fermentativo da matéria-prima. No caso das amostras exploradas neste trabalho, este efeito é minimizado, pois a mesma cachaça foi utilizada no preparo de todos os extratos. Assim, as diferenças entre os espectros eletrônicos de absorção aqui relatadas devem ser atribuídas somente aos componentes extratíveis de cada espécie de madeira. A Figura 1 apresenta a titulo de ilustração a derivada primeira dos espectros obtidos para os extratos de carvalho e de jatobá (A) e os cromatogramas dos extratos correspondentes (B).
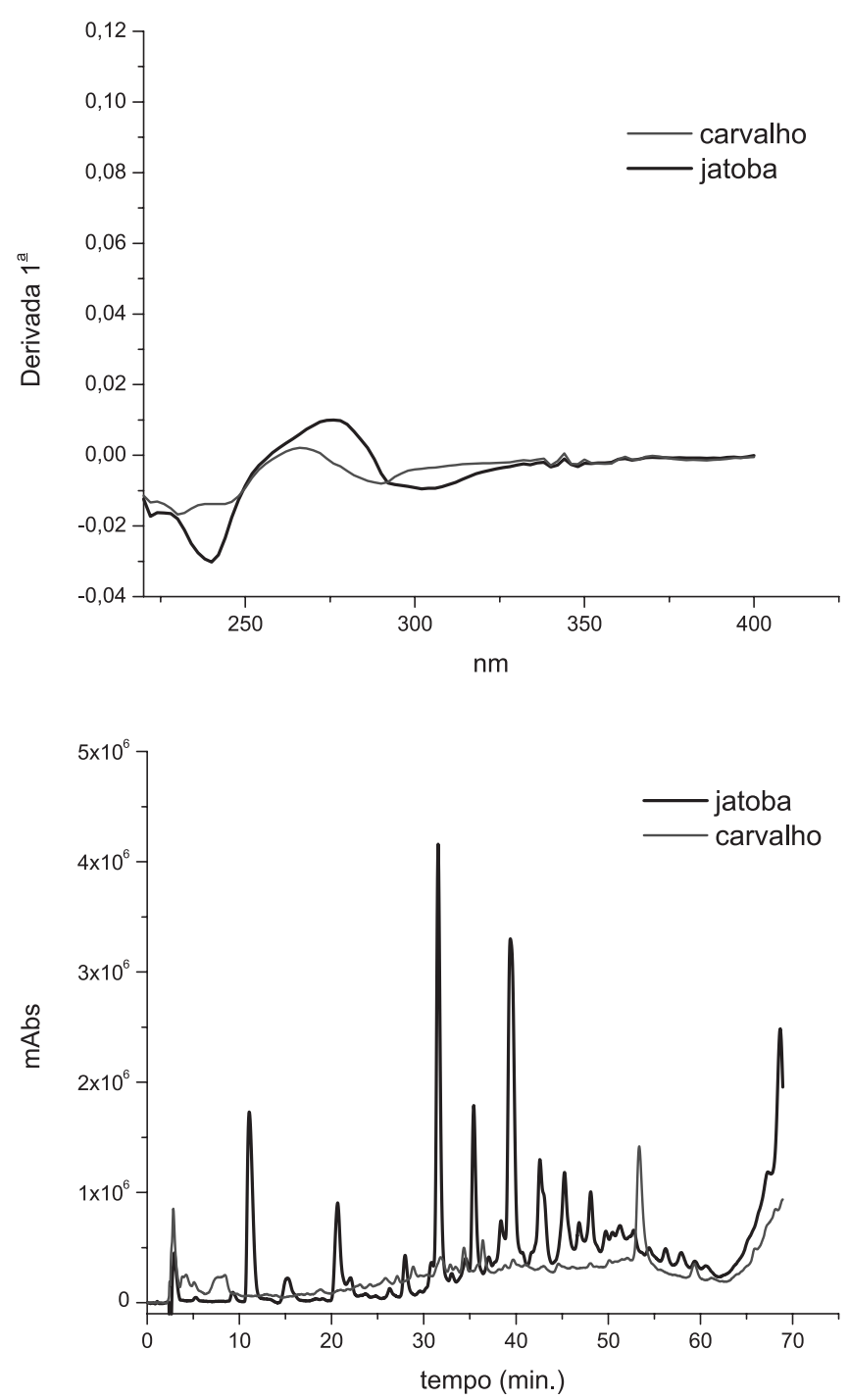

Figura 1. Gráficos da primeira derivada aplicada ao espectro eletrônico de amostras de carvalho e jatobá (1 A) e cromatogramas das mesmas amostras, com o pico identificado como cumarina no extrato de jatobá no tempo de retenção $\left(t_{R}\right)=39,4$ '

Os conjuntos de dados espectrofotométricos de absorbância, obtidos da primeira derivada dos espectros eletrônicos dos extratos, e dados cromatográficos originados dos cromatogramas, estes últimos reportados anteriormente, ${ }^{16}$ foram analisados individualmente por análise de componentes principais (ACP). No caso dos espectros eletrônicos de absorção, foram analisados valores de absorbância versus comprimentos de onda; em se tratando dos cromatogramas os parâmetros foram tempos de retenção e absorbância a $280 \mathrm{~nm}$. Os resultados desta análise indicaram em ambos os casos a formação de 5 grupos, um para cada tipo de extrato de madeira. $\mathrm{Na}$ análise dos dados obtidos por espectrofotometria os valores das duas primeiras 
CPs explicam $91 \%$ da variância do conjunto de dados originais (CP1 $81 \%+\mathrm{CP} 210 \%$ da Figura 2) enquanto que os dados gerados a partir dos cromatogramas permitem explicar $65 \%$ dos dados de variância (CP1 52\% + CP2 13\% da Figura 1S, material suplementar).
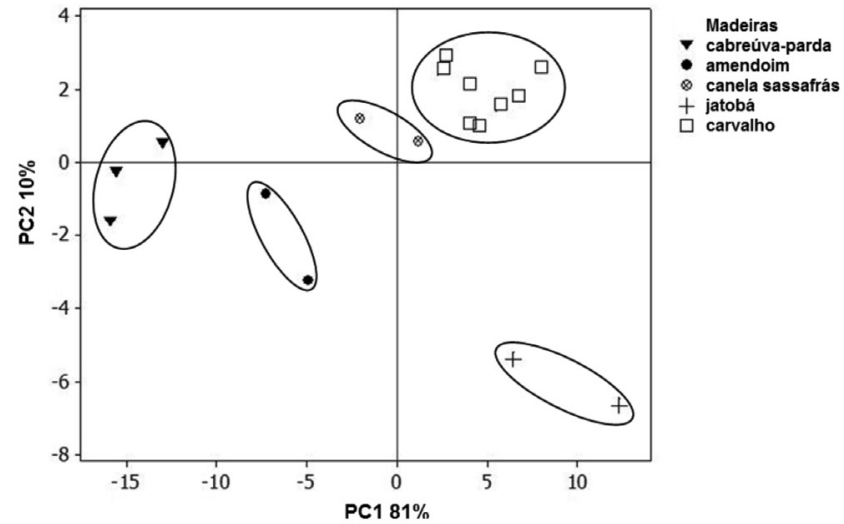

Figura 2. Gráfico de scores obtidos dos espectros eletrônicos na região do $U V$ dos extratos de madeiras

Embora o fingerprint cromatográfico apresente um número maior de informações sobre a composição química dos extratos ${ }^{16}$ do que o espectro eletrônico, na presente comparação esta riqueza não se refletiu positivamente na explicação da variância dos dados originais.

Em se tratando dos resultados cromatográficos, os gráficos de loading (loading versus tempo de retenção) indicaram ${ }^{16}$ quais os picos mais relevantes para cada madeira. O uso de métodos cromatográfícos ${ }^{16}$ associados à espectrometria de massas possibilitou confirmar e quantificar os compostos correspondentes a estes picos. Assim, por exemplo, a cumarina, presente em extratos de jatobá em teores $\left(\mathrm{mg} \mathrm{L}^{-1}\right) 10$ vezes superiores aos das demais madeiras, foi determinante na distinção deste extrato dentre os demais. ${ }^{16}$ Os baixos teores de cumarina e de escopoletina $\left(\leq 0,002 \mathrm{mg} \mathrm{L}^{-1}\right.$ para ambas) em $63 \%$ das amostras de carvalho analisadas e os elevados teores de ácido elágico $\left(\geq 0,009 \mathrm{mg} \mathrm{L}^{-1}\right)$ foram importantes para a caracterização do agrupamento constituído pelas amostras de carvalho. ${ }^{25}$ Para as amostras de canela sassafrás os picos atribuídos aos compostos 5-hidroximetil-furfuraldeído (5HMF) e furfuraldeído (HF) aparecem como os mais relevantes na $\mathrm{CP} 2$, atuando decisivamente para a distinção desta madeira. ${ }^{16}$

Processos de degradação de carboidratos são conhecidas fontes de compostos furânicos como furfural, 5-metilfurfural e 5-hidroximetilfurfural, provenientes das degradações via reação de Maillard da pentose, raminose e hexose, respectivamente, açúcares presentes em madeiras. ${ }^{26}$ É interessante ressaltar que furfural e 5-hidroximetilfurfural (5-HMF, HF) são controlados pela Instrução Normativa 13. ${ }^{8}$ Portanto, a contribuição da extração de 5-hidroximetilfurfural e furfural da madeira pelo destilado e seu aporte ao teor de aldeído totais deve ser considerada no processo de seleção da espécie e tratamento térmico da madeira a ser utilizada na construção do tonel.

Conforme já mencionado, na exploração dos dados espectrais (UV) gerados pelos valores de absorbância dos extratos, visivelmente os gráficos de ACP (Figura 2) apresentam maior poder de discriminação do que os provenientes da análise cromatográfica (Figura 1S, material suplementar). Na análise da primeira componente (CP 1) observa-se a definição de dois grupos: um situado nos valores positivos do gráfico de scores e outro nos valores negativos (Figura 2).

A análise conjunta dos gráficos de loading (Figura 3) e de scores sugere que os valores de absôrbancia próximos a $280 \mathrm{~nm}$ são os mais correlacionados com a formação dos grupos situados à direita do gráfico da Figura 2.

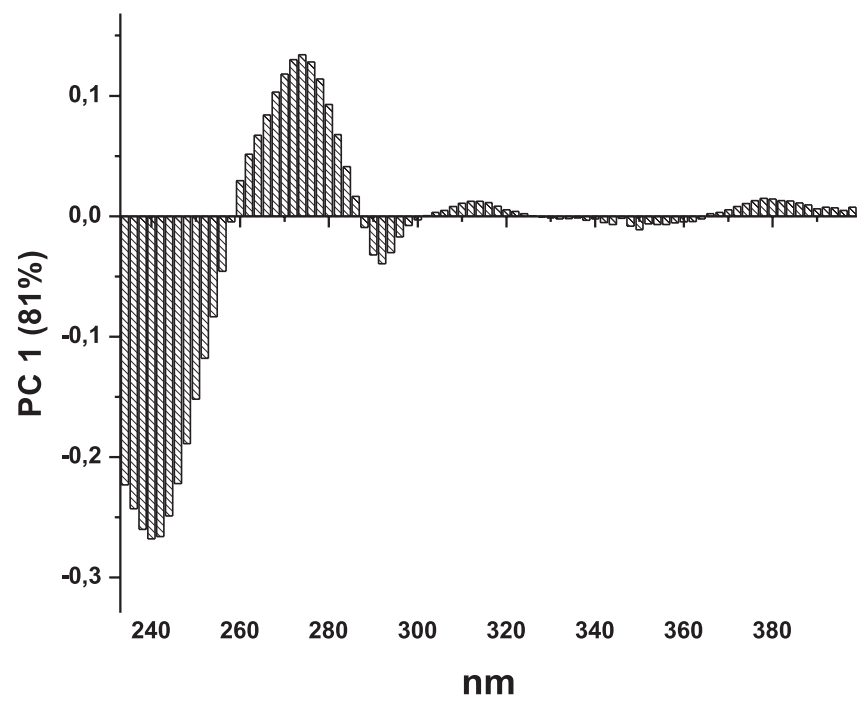

Figura 3. Gráficos de loading para a PC1 dos espectros eletrônicos de UV mostrando os comprimentos de ondas ( $\mathrm{nm}$ ) com maiores pesos na separação

Estes correspondem às amostras dos extratos de carvalho e jatobá. Compostos fenólicos em sua maioria apresentam banda de absorção máxima próxima a $280 \mathrm{~nm}$. A análise de fenóis totais (método de Folin-Ciocalteu) indica que os extratos de carvalho e jatobá apresentaram, dentre os diferentes extratos estudados, ${ }^{12,16}$ os maiores teores destes compostos. Este fato certamente influenciou nos espectros eletrônicos e, portanto, na definição dos clusters observados.

Os baixos teores de vanilina $\left(\lambda_{\max }=232\right.$ e $\left.309 \mathrm{~nm}\right)$ e o elevado teor de cumarina $\left(\lambda_{\max }=280 \mathrm{~nm}\right)$ em extratos de jatobá exemplificam esta tendência. ${ }^{16} \mathrm{O}$ mesmo se observa com respeito à presença de ácido elágico $\left(\lambda_{\max }=280 \mathrm{~nm}\right)$ nas amostras de carvalho.

À esquerda do gráfico de scores (valores negativos) estão os extratos de amendoim e de cabreúva-parda. Estes, apesar de também absorverem a $280 \mathrm{~nm}$, possuem seu posicionamento na Figura 1 dependente das absorbâncias próximas a $230 \mathrm{~nm}$. Nas amostras em questão, a absorção nesta região do espectro é atribuída majoritariamente à presença de compostos não fenólicos, tais como HF $\lambda_{\text {max }}=233$ e $276 \mathrm{~nm} .{ }^{16,27}$ Também contribuem para a absorção nesta região outros compostos passíveis de serem extraídos em seu contato com madeiras, que apresentem bandas com máximos próximos à região citada, tais como a vanilina $\lambda_{\max }=238$ e $315 \mathrm{~nm}$, o seringaldeído $\lambda_{\max }=238$ e $309 \mathrm{~nm}$. Esta informação está de acordo com o observado a partir da análise dos perfis cromatográficos, conforme apresentado anteriormente neste trabalho.

O espectro eletrônico de absorção é uma somatória das contribuições das diversas transições eletrônicas e, portanto, trata-se de uma resultante muitas vezes bastante complexa. Madeiras como a cabreúva-parda e o amendoim, cujos agrupamentos se apresentam fortemente correlacionados com as absorbâncias próximas a 230 nm, apresentam elevados teores de aldeídos cinâmicos, metabólitos secundários dos vegetais, em sua composição química. ${ }^{16}$ Tais aldeídos, como sinapaldeído e coniferaldeído possuem em seus perfis espectrais duas bandas com máximos próximos a 240 e 309 nm.

A comparação entre as duas metodologias aponta, com base na explicação dos valores de variância, que o modelo obtido a partir dos espectros de UV foi o mais eficiente na análise exploratória para a separação dos extratos de madeiras. Esta métodologia tem por virtude características como simplicidade, baixo custo e rapidez, envolvendo instrumentação extensamente difundida, robusta, de fácil manutenção e, portanto, presente mesmo nos laboratórios mais simples. Desta forma candidata-se como uma opção bastante acessível e adequada 
para a análise de rotina de bebidas e de extratos de madeiras.

Como teste preliminar, foram obtidos espectros eletrônicos de algumas cachaças envelhecidas em carvalho, jatobá, amendoim, cabreúva e canela sassafrás. Os resultados experimentais correspondentes foram tratados de forma semelhante aos obtidos para os extratos. Nota-se, na Figura 4, que as cachaças se agrupam juntamente com os extratos das madeiras correspondentes.

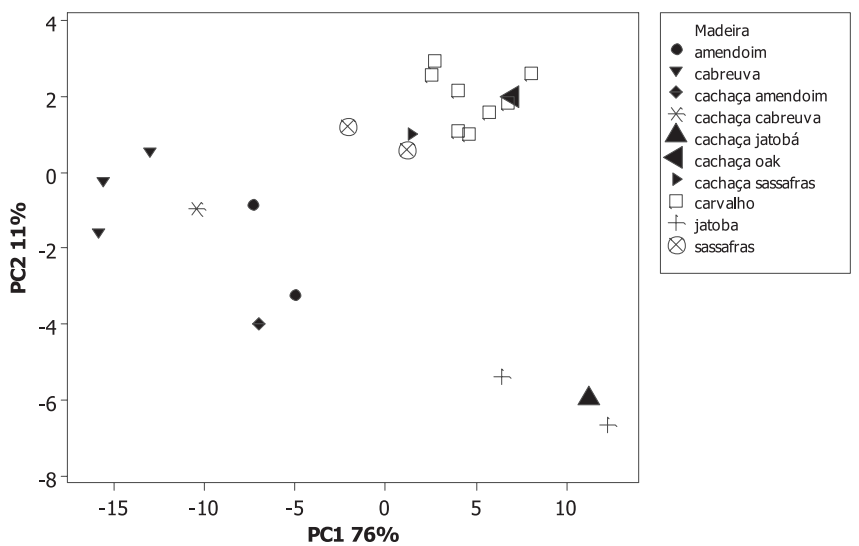

Figura 4. Gráfico de scores obtido a partir dos espectros eletrônicos dos extratos de madeiras e cachaças comerciais

\section{CONCLUSÃO}

Os dados cromatográficos explorados por ACP, embora apresentando uma menor eficiência na explicação dos dados de variância, permitem através do tratamento quimiométrico identificar quais compostos são os mais prováveis candidatos a marcadores quími$\cos$, fato este de por si relevante para o conhecimento da química de bebidas envelhecidas.

Amostras de canela sassafrás, madeira esta controlada pelo IBAMA e cujo uso é desaconselhado para a confecção de tonéis, ${ }^{12-14}$ foram utilizadas procurando fornecer subsídios para elaboração de uma metodologia para a identificação de seus extratos.

Dados sobre a presença de furfuraldeído em canela sassafrás, aldeídos cinâmicos em cabreúva-parda podem se constituir em parâmetros a serem controlados. O primeiro em função da sua presença indesejável ${ }^{8,28}$ e o segundo, devido à sua apreciação sensorial positiva em bebidas de alta qualidade. ${ }^{3-5}$ Aspectos ligados às possibilidades de aplicações destas metodologias em taxonomia vegetal estão sendo investigados e os resultados serão publicados posteriormente.

\section{MATERIAL SUPLEMENTAR}

No material suplementar, disponível gratuitamente em http:// quimicanova.sbq.org.br, na forma de aquivo PDF, encontram-se a Figura 1S (Gráfico de score obtido por tratamento dos dados cromatográficos (CLAE-DAD)) e as condições cromatográficas empregadas nesta análise.

\section{AGRADECIMENTOS}

Ao CNPq, à Capes e FAPESP pelo auxílio financeiro e aos Profs. F. A. R. Lahr e J. R. Piggot pelas amostras de madeiras.

\section{REFERÊNCIAS}

1. Cardoso, D. R.; Andrade Sobrinho, L. G.; Lima-Neto, B. S.; Franco, D. W.; J. Braz. Chem. Soc. 2004, 15, 277.

2. Arfelli, G.; Sartini, E.; Corzani, C.; Fabiani, A.; Natali, N.; Food Sci. Technol. Int. 2007, 1, 293.

3. Lee, M.; Paterson, A.; Piggott, J. R.; J. Inst. Brew. 2001, 107, 5.

4. Mosedale, J. R.; Forestry 1995, 68, 203.

5. Nishimura, K.; Matsuyama, R. Em Maturation and Maturation Chemistry; Piggot, J. R., ed.; Longman: London, 1989, cap. 7.

6. União Europeia, Regulation No 110/2008 of the European Parliament and of the Council; Official Journal of the European Union 13/02/2008.

7. Lima, U. A.; Aguardente: fabricação em pequenas destilarias, Ed. FEALQ: Piracicaba, 1999.

8. Brasil, Ministério da Agricultura Pecuária e Abastecimento; Instrução Normativa $\mathrm{n}^{\circ}$ 13, de 29/6/2005, Diário Oficial da União, 30/06/2005, seção 1, p. 3 .

9. Faria, J. B.; Cardello, H. M. A. B; Boscolo, M.; Isique, W. D; Odello, L.; Franco, D. W.; Eur. Food Res. Technol. 2003, $218,83$.

10. Boscolo, M.; Andrade-Sobrinho, L. G.; Lima-Neto, B. S.; Ferreira, M. M. C.; Franco, D. W.; J. AOAC Int. 2002, 85, 744.

11. Fregoni, M.; Professor Doutor de Viticoltura e Diretor do Istituto di Frutti-Viticoltura, Italia, comunicação pessoal.

12. Hagan, E. C.; Jenner, P. M.; Jones, W. I.; Fitzhuch, O. G.; Long, E. L.; Brouwer, J. G.; Webb, W. K.; Toxicol. Appl. Pharmacol. 1965, 7, 18.

13. Weisburger, E. K.; J. Chem. Educ. 1980, 57, 724.

14. Cardoso, D. R.; Frederiksen, A. M.; Da Silva, A. A.; Franco, D. W.; Skibsted, L. H.; Eur. Food Res. Technol. 2008, 227, 1109.

15. Alcarde, A. R.; Souza, P. A; Belluco, A. E. S.; Ciênc. Tecnol. Aliment. 2010, 30, 1

16. Da Silva, A. A.; Nascimento, E. S. P.; Cardoso, D. R.; Franco, D. W.; J. Sep. Sci. 2009, 32, 3681.

17. De Souza, P. P.; Siebald, H. G. L.; Augusti, D. V.; Borges-Neto, W.; Amorim, V. M.; Catharino, R. R.; Eberlin, M. N.; Augusti, R.; J. Agric. Food Chem. 2007, 55, 2094.

18. Odello, L.; Braceschi, G. P.; Seixas, F. R. F.; Da Silva, A. A.; Galinar, C. A.; Franco, D. W.; Quim. Nova 2009, 32, 1839.

19. Bettin, S. M.; Isique, W. D.; Franco, D. W.; Andersen, M. L.; Knudsen, S.; Skibsted, L. H.; Eur. Food Res. Technol. 2002, 215, 169.

20. Vicente, C. D.; De Abreu, F. C.; Goulart, M. O. F.; De Vasconcelo, J. N.; Am. J. Food Technol. 2011, 6, 631.

21. http://www.ebah.com.br/content/ABAAABt68AI/identificao-madeirasipt.pdf, acessada em Junho 2011.

22. Teixeira, V. L.; Kelecom, A.; Gottlieb, O. R.; Quim. Nova 1990, 13, 293.

23. Skov, T.; van den Berg, F.; Tomasi, G.; Bro, R.; J. Chemom. 2006, 20, 484.

24. Barbosa-García, O.; Ramos-Ortíza, G.; Maldonado, J. L.; PichardoMolina, J. L.; Meneses-Nava, M. A.; Landgrave, J. E.; CervantesMartínez, J.; Spectrochim. Acta, Part A 2007, 66, 129.

25. Viriot, C.; Scalbert, A.; Lapierre, C.; Moutounets, M.; J. Agric. Food Chem. 1993, 41, 1872.

26. Villalón Mir, M.; López, H.; De La Serrana, G.; Martínez, C. L; Granados, J. Q.; J. Liq. Chromatogr. 1991, 14, 3615.

27. Nascimento, R. F.; Marques, J. C.; Keukeleire, D.; Lima-Neto, B. S.; Franco, D. W.; J. Chromatogr., A 1997, 782, 13.

28. Arfelli, G.; Sartini, E.; Corzani, C.; Fabiani, A.; Natali, N.; Food Sci. Technol. Int. 2007, 13, 293. 


\section{IDENTIFICAÇÃO DE EXTRATOS ETANÓLICOS DE MADEIRAS UTILIZANDO SEU ESPECTRO ELETRÔNICO DE ABSORÇÃO E ANÁLISE MULTIVARIADA}

Alexandre Ataide da Silva, Eduardo Sanches Pereira do Nascimento, Daniel Rodrigues Cardoso e Douglas Wagner Franco* Instituto de Química de São Carlos, Universidade de São Paulo, CP 780, 13560-970 São Carlos - SP, Brasil

\section{Condições cromatográficas}

Os cromatogramas foram obtidos por injeção direta da amostra em cromatógrafo a líquido Shimadzu modelo $10 \mathrm{AD}$ equipado com um detector de arranjo de diodo modelo SPDM6A, operando em $280 \mathrm{~nm}$, utilizando uma coluna C-18 Shimpack com dimensões de $2 \mathrm{~mm}$ x $250 \mathrm{~mm}$ x 4,6 $\mu \mathrm{m}$, acoplada a uma pré-coluna Shim-pack CLC G-ODS ( 30 x 4,6 mm). O injetor utilizado foi Rheodyne modelo $7725 \mathrm{i}$, com alça de amostragem de $5 \mu \mathrm{L}$. A fase móvel com vazão de 0,4 mL/min consistia em uma mistura de solventes: A (água/ácido acético, \% 98:2 v/v) e B (metanol/água/ácido acético, 70:28:2\% v/v), utilizando gradiente de eluição com a seguinte programação: 0-3 min: B 0\% em A; 3-25 min: B 40\% em A, 25-43 min: B 60\% em A, 43-55 min: B 60\% em A, 55-60 min: 80\% B em A; 60-65 min: $80 \%$ B em A; e 65-69min: 0\% B.

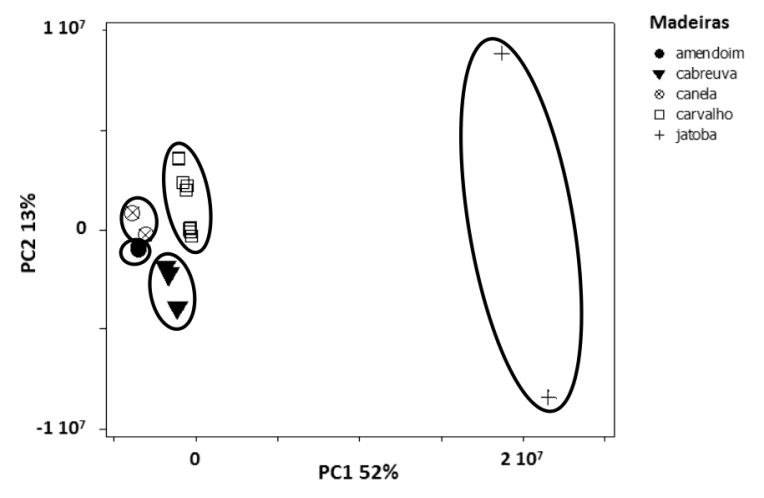

Figura 1S. Gráfico de scores para os dados obtidos por CLAE-DAD (280 nm) dos extratos de madeiras 